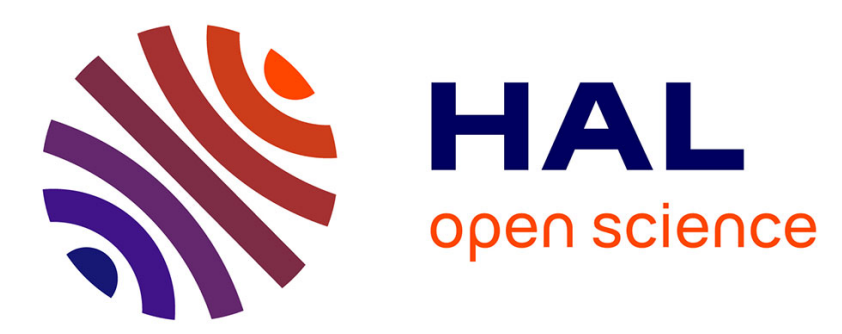

\title{
A fuzzy adaptive variable-structure control scheme for uncertain chaotic MIMO systems with sector nonlinearities and dead-zones
}

\author{
Abdesselem Boulkroune, Mohammed M’Saad
}

\section{- To cite this version:}

Abdesselem Boulkroune, Mohammed M'Saad. A fuzzy adaptive variable-structure control scheme for uncertain chaotic MIMO systems with sector nonlinearities and dead-zones. Expert Systems with Applications, 2011, 38 (12), pp.Pages 14744-14750. 10.1016/j.eswa.2011.05.006 . hal-01060343

\section{HAL Id: hal-01060343 \\ https://hal.science/hal-01060343}

Submitted on 9 Sep 2014

HAL is a multi-disciplinary open access archive for the deposit and dissemination of scientific research documents, whether they are published or not. The documents may come from teaching and research institutions in France or abroad, or from public or private research centers.
L'archive ouverte pluridisciplinaire HAL, est destinée au dépôt et à la diffusion de documents scientifiques de niveau recherche, publiés ou non, émanant des établissements d'enseignement et de recherche français ou étrangers, des laboratoires publics ou privés. 


\title{
A fuzzy adaptive variable-structure control scheme for uncertain chaotic MIMO systems with sector nonlinearities and dead-zones
}

\author{
A. Boulkroune ${ }^{\mathrm{a}, *}$, M. M'Saad $^{\mathrm{b}}$ \\ ${ }^{a}$ Department of Automatic, Faculty of Engineering Sciences, University of Jijel, BP. 98, OuledAissa, 18000 Jijel, Algeria \\ ${ }^{\mathrm{b}}$ GREYC, UMR 6072 CNRS, Université de Caen, ENSICAEN, 6 Bd Maréchal Juin, 14050 Caen Cedex, France
}

Keywords:

Adaptive control

Fuzzy system

Variable-structure control

MIMO chaotic systems

Sector nonlinearities

Dead-zone

\begin{abstract}
A B S T R A C T
In this paper, a fuzzy adaptive variable-structure controller is investigated for a class of uncertain multiinput multi-output (MIMO) chaotic systems with both sector nonlinearities and dead-zones. A suitable adaptive fuzzy system is used to reasonably approximate the uncertain functions. A Lyapunov approach is employed to derive the parameter adaptation laws and prove the boundedness of all signals of the closed-loop system as well as the exponential convergence of the closed-loop errors to an adjustable region. The proposed controller can be applied to the systems with or without sector nonlinearities and/or dead-zones in the input. The effectiveness of the proposed fuzzy adaptive controller is illustrated throughout simulation results.
\end{abstract}

\section{Introduction}

Chaotic system is a very complex dynamical nonlinear system and its response exhibits some specific features such as excessive sensitivity to initial conditions, broad Fourier transform spectrums, and irregular identities of the motion in phase plane (Chen \& Ueta, 1999; Vanecek \& Celikovsky, 1996). Chaos control problem was firstly considered by Ott, Grebogi, and Yorke (1990). Since then, it has been extensively investigated in the past two decades (Chen \& Dong, 1998). Several (linear and nonlinear) control techniques have been successfully applied for the control of chaotic systems (for synchronisation, tracking, or stabilisation purposes) such that: PID control (Ghezi \& Peccardi, 1997), adaptive feedback control (Feki, 2003; Hua, Guan, \& Shi, 2005), observer-based control (Boulkroune, Chekireb, Tadjine, \& Bouatmane, 2006; Boulkroune, Chekireb, Tadjine, \& M'Saad, 2006; Boulkroune, Chekireb, Tadjine, \& Bouatmane, 2007), sliding-mode control (Ablay, 2009; Nazzal \& Natsheh, 2007; Yau, Chen, \& Chen, 2000), adaptive backstepping control (Ge \& Wang, 2000; Wang \& Ge, 2001), adaptive fuzzy control (Boulkroune et al., 2006; Chang, 2001; Liu \& Zheng, 2009; Poursamad \& Markazi, 2009; Roopaei \& Jahromi, 2008; Roopaei, Jahromi, \& Jafari, 2009), adaptive neural control (Ge \& Wang, 2002), etc. A key assumption in all previous control schemes (Ablay, 2009; Boulkroune, Chekireb, Tadjine, \& Bouatmane, 2006; Boulkroune, Chekireb, Tadjine, \& M'Saad, 2006; Boulkroune et al., 2007; Chang, 2001; Feki, 2003; Ghezi and Peccardi, 1997; Ge and

\footnotetext{
* Corresponding author.

E-mail addresses: boulkroune2002@yahoo.fr (A. Boulkroune), msaad@greyc.en sicaen.fr (M. M'Saad).
}

Wang, 2000, 2002; Hua et al., 2005; Liu and Zheng, 2009; Nazzal and Natsheh, 2007; Poursamad and Markazi, 2009; Roopaei and Jahromi, 2008; Roopaei et al., 2009; Wang and Ge, 2001; Yau et al., 2000) is that the chaotic system has linear inputs.

The control problem of uncertain nonlinear (chaotic or nonchaotic) systems with nonlinear inputs has received a great interest because of the input nonlinearities, such as saturation, backlash, dead-zones, and so on, naturally originate from physical limitations in system realization (Boulkroune, M'Saad, Tadjine, \& Farza, 2008; Hsu, Wang, \& Lin, 2004). It is worth mentioning that the existence of input nonlinearities may leads to notable performance degradations or even instability of the control system. It is thereby more advisable to take into account the effects of the input nonlinearities in the control design as well as the stability analysis. Recently, some control schemes have been proposed (Chang, 2007; Chiang, Hung, Yan, Yang, \& Chang, 2007; Hung, Yan, \& Liao, 2008; Yan, Shyu, \& Lin, 2005) for a class of chaotic systems with input sector nonlinearities and/or dead-zones. However, these underlying results suffer from some fundamental limitations. Firstly, the class considered of the chaotic systems is relatively simple (i.e. the chaotic systems considered in these works is not of type MIMO). Secondly, the so-called gain reduction tolerances of the input nonlinearities and upper bounds of the model uncertainties are required to be known or partially known.

Motived by works in Yan et al. (2005), Chiang et al. (2007), Chang (2007) and Hung et al. (2008), one aims at designing a fuzzy adaptive variable-structure controller for a class of uncertain chaotic MIMO systems containing both sector nonlinearities and deadzones. Bearing in mind the available results (Chang, 2007; Chiang et al., 2007; Hung et al., 2008; Yan et al., 2005), the main contributions of this paper are: 
- The class considered of the chaotic systems is MIMO.

- The knowledge of gain reduction tolerances of the nonlinear dead-zones is not necessary in the controller design.

- The upper bounds of the uncertainties (i.e. nonlinear functions) are assumed to be unknown and estimated by using adaptive fuzzy systems.

\section{Notation, problem statement and preliminaries}

Consider the following class of uncertain chaotic MIMO systems described by:

$$
\begin{aligned}
& y_{1}^{\left(r_{1}\right)}=f_{1}(x)+\sum_{j=1}^{p}\left(g_{1 j} \Phi_{j}\left(u_{j}\right)\right)+d_{1}(t), \\
& \vdots \\
& y_{p}^{\left(r_{p}\right)}=f_{p}(x)+\sum_{j=1}^{p}\left(g_{p j} \Phi_{j}\left(u_{j}\right)\right)+d_{p}(t),
\end{aligned}
$$

where $x=\left[y_{1}, \dot{y}_{1}, \ldots, y_{1}^{\left(r_{1}-1\right)}, \ldots, y_{p}, \dot{y}_{p}, \ldots, y_{p}^{\left(r_{p}-1\right)}\right]^{T} \in R^{r}$ is the overall state vector which is assumed to be available for measurement and, $u=\left[u_{1}, \ldots, u_{p}\right]^{T} \in R^{p}$ is the control input vector, $y=\left[y_{1}, \ldots, y_{p}\right]^{T} \in R^{p}$ is the output vector, and $f_{i}(x), i=1, \ldots, p$ are unknown continuous nonlinear functions, $g_{i j}, i, j=1, \ldots, p$ are unknown constant control-gains, $\Phi(u)=\left[\Phi_{1}\left(u_{1}\right), \Phi_{2}\left(u_{2}\right), \ldots, \Phi_{p}\left(u_{p}\right)\right]^{T}$ is a nonlinear input function vector satisfying some properties which will be given later, and $d_{i}(t)$ are unknown external disturbances.

Let us denote

$$
\begin{gathered}
y^{(r)}=\left[y_{1}^{\left(r_{1}\right)} \ldots y_{p}^{\left(r_{p}\right)}\right]^{T}, \quad F(x)=\left[f_{1}(x) \ldots f_{p}(x)\right]^{T}, \\
D(t)=\left[d_{1}(t) \ldots d_{p}(t)\right]^{T}, \quad G=\left[\begin{array}{ccc}
g_{11} & \ldots & g_{1 p} \\
\vdots & \ddots & \vdots \\
g_{p 1} & \ldots & g_{p p}
\end{array}\right] .
\end{gathered}
$$

Then, the system (1) can be rewritten in the following compact form:

$y^{(r)}=F(x)+G \Phi(u)+D(t)$,

where $F(),. \Phi(),. D(.) \in R^{p}$ and $G(.) \in R^{p \times p}$.

Now, we make the following reasonable assumptions.

Assumption 1 The desired trajectory vector $x_{d}=\left[x_{d 1}^{T}, \ldots, x_{d p}^{T}\right.$, $\left.y_{d 1}^{\left(r_{1}\right)}, \ldots, y_{d p}^{\left(r_{p}\right)}\right]$, where $x_{d i}=\left[y_{d i}, \dot{y}_{d i}, \ldots, y_{d i}^{\left(r_{i}-1\right)}\right]^{T}, \forall i=1, \ldots, p$, is supposed to be continuous, bounded and available for measurement. Then, $x_{d} \in \Omega_{x_{d}} \subset R^{r+p}$, with $\Omega_{x_{d}}$ is a known compact set.

\section{Assumption 2}

(a) Without loss of generality, we assume that the unknown control-gains matrix $G$ is symmetric and positive-definite. As a result, there exists an unknown positive constant $\sigma_{g 0}$, such that: $G \geqslant \sigma_{g 0} I_{p}$, with $I_{P}$ is the $p \times p$ identity matrix.

(b) The disturbance $d_{i}(t)$ is bounded as follows: $\left|d_{i}(t)\right| \leqslant$ $\bar{d}_{i}, \forall i=1, \ldots, p$, with $\bar{d}_{i}$ is unknown positive constant.

\section{Remark 1}

(a) The model (2) can be used to describe a large class of MIMO or SISO (chaotic or non chaotic) nonlinear dynamical systems namely:

- Robotic systems: e.g. single-link robot, two-link robotic manipulator used in Kar and Behera (2009),
- Induction motors (Youcef \& Wahba, 2009),

- Some mechanical systems: e.g. mass-spring-damper system in Chen, Lin, and Chen (2008),

- Chaotic systems: e.g. Chua's circuit (Chang, 2001), Unified chaotic system (Lü, Chen, Cheng, \& Celikovssky, 2002; Ablay, 2009), Arneodo chaotic system (Hua et al., 2005), gyro system (Roopaei et al., 2009), Genesio chaotic system, Rössler system, Duffing and Holmes system, and many others.

(b) Assumption 2a is not restrictive, as it is satisfied by many physical systems. Note that this assumption is frequent in the literature devoted to adaptive (fuzzy or neural) control of MIMO systems. Also, it guarantees the controllability of the system (1).

(c) Note that the fundamental results of this paper can be adapted, with few modifications, to the case where $G$ is symmetric and negative-definite.

The objective of this paper is to design a control law $u$ such as the output vector $y$ follows the specified desired trajectory $y_{d}=\left[y_{d 1}, \ldots, y_{d p}\right]^{T} \in R^{p}$, with all involved signals in the closed-loop system remain bounded.

Let us define the tracking error by

$$
\begin{gathered}
e_{1}=y_{1}-y_{d 1}, \\
\vdots \\
e_{p}=y_{p}-y_{d p}
\end{gathered}
$$

and the filtered tracking error as

$S=\left[S_{1}, \ldots, S_{p}\right]^{T}$

with

$S_{i}=\left[\frac{d}{d t}+\lambda_{i}\right]^{r_{i}-1} e_{i}, \quad$ for $\quad \lambda_{i}>0, \quad \forall i=1, \ldots, p$.

Then, we can write (5) as follows

$S_{i}=\lambda_{i}^{r_{i}-1} e_{i}+\left(r_{i}-1\right) \lambda_{i}^{r_{i}-2} \dot{e}_{i}+\cdots+\left(r_{i}-1\right) \lambda_{i} e_{i}^{\left(r_{i}-2\right)}+e_{i}^{\left(r_{i}-1\right)}$,

with $i=1, \ldots, p$.

Notice that if we choose $\lambda_{i}>0$, with $i=1, \ldots, p$, then the roots of the polynomial $H_{i}(s)=\lambda_{i}^{r_{i}-1}+\left(r_{i}-1\right) \lambda_{i}^{r_{i}-2} s+\cdots+\left(r_{i}-1\right) \lambda_{i} s^{r_{i}-2}+$ $s^{r_{i}-1}$ related to the characteristic equation of $S_{i}=0$ are all in the open left-half plane.

The relation (6) can be rewritten in the following compact form

$S_{i}=C_{i}^{T} E_{i}$

with

$E_{i}=\left[\begin{array}{lllll}e_{i} & \dot{e}_{i} & \cdots & e_{i}^{\left(r_{i}-2\right)} & e_{i}^{\left(r_{i}-1\right)}\end{array}\right]^{T}$,

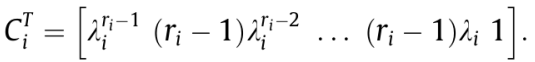

Consequently, the vector $S$ takes the form:

$S=C^{T} E$

where

$C^{T}=\operatorname{diag}\left[C_{1}^{T} C_{2}^{T} \ldots C_{p}^{T}\right]_{(p \times r)}$,

$E=\left[\begin{array}{llll}E_{1}^{T} & E_{2}^{T} & \ldots & E_{p}^{T}\end{array}\right]_{(r \times \mathbf{1})}^{T}$.

And the dynamic of $S_{i}$ is described by:

$\dot{S}_{i}=C_{r i}^{T} E_{i}+e_{i}^{\left(r_{i}\right)}, \quad$ and $i=1, \ldots, p$, 
where $C_{r i}$ is given by

$C_{r i}^{T}=\left[0 \lambda_{i}^{r_{i}-1}\left(r_{i}-1\right) \lambda_{i}^{r_{i}-2} \ldots 0.5\left(r_{i}-1\right)\left(r_{i}-2\right) \lambda_{i}^{2}\left(r_{i}-1\right) \lambda_{i}\right]$.

The dynamic of $S$ can be written in the following compact form

$\dot{S}=C_{r}^{T} E+e^{(r)}$,

where

$C_{r}^{T}=\operatorname{diag}\left[\begin{array}{llll}C_{r 1}^{T} & C_{r 2}^{T} & \cdots & C_{r p}^{T}\end{array}\right]_{(p \times r)}$,

$e^{(r)}=\left[\begin{array}{llll}e_{1}^{\left(r_{1}\right)} & e_{2}^{\left(r_{2}\right)} & \cdots & e_{p}^{\left(r_{p}\right)}\end{array}\right]^{T}$

with

$e^{(r)}=y^{(r)}-y_{d}^{(r)}$,

where $y^{(r)}=\left[\begin{array}{llll}y_{1}^{\left(r_{1}\right)} & y_{2}^{\left(r_{2}\right)} \cdots & y_{p}^{\left(r_{p}\right)}\end{array}\right]^{T}$ is previously defined, and

$y_{d}^{(r)}=\left[\begin{array}{llll}y_{d 1}^{\left(r_{1}\right)} & y_{d 2}^{\left(r_{2}\right)} & \cdots & y_{d p}^{\left(r_{p}\right)}\end{array}\right]^{T}$,

From (18), we can write (15) as follows

$\dot{S}=C_{r}^{T} E+y^{(r)}-y_{d}^{(r)}$.

Thereafter, (20) will be used in the development of the proposed controller and the stability analysis.

\subsection{Description of the fuzzy logic system}

The basic configuration of a fuzzy logic system consists of a fuzzifier, some fuzzy IF-THEN rules, a fuzzy inference engine and a defuzzifier, as shown in Fig. 1. The fuzzy inference engine uses the fuzzy IF-THEN rules to perform a mapping from an input vector $\underline{x}^{T}=\left[x_{1}, x_{2}, \ldots, x_{n}\right] \in R^{n}$ to an output $\hat{f} \in R$.

The $i$ th fuzzy rule is written as

$R^{(i)}: \quad$ if $x_{1}$ is $A_{1}^{i}$ and $\ldots$ and $x_{n}$ is $A_{n}^{i}$ then $\hat{f}$ is $f^{i}$,

where $A_{1}^{i}, A_{2}^{i}, \ldots$, and $A_{n}^{i}$ are fuzzy sets and $f^{i}$ is the fuzzy singleton for the output in the ith rule. By using the singleton fuzzifier, product inference, and center-average defuzzifier, the output of the fuzzy system can be expressed as follows:

$\hat{f}(\underline{x})=\frac{\sum_{i=1}^{m} f^{i}\left(\prod_{j=1}^{n} \mu_{A_{j}^{i}}\left(x_{j}\right)\right)}{\sum_{i=1}^{m}\left(\prod_{j=1}^{n} \mu_{A_{j}^{i}}\left(x_{j}\right)\right)}=\theta^{T} \psi(\underline{x})$,

where $\mu_{A^{i}}\left(x_{j}\right)$ is the degree of membership of $x_{j}$ to $A_{j}^{i}, m$ is the number of fuzzy rules, $\theta^{T}=\left[f^{1}, f^{2}, \ldots, f^{m}\right]$ is the adjustable parameter vector (composed of consequent parameters), and $\psi^{T}=\left[\psi^{1}, \psi^{2}, \ldots, \psi^{m}\right]$, with

$\psi^{i}(\underline{x})=\frac{\left(\prod_{j=1}^{n} \mu_{A_{j}^{i}}\left(x_{j}\right)\right)}{\sum_{i=1}^{m}\left(\prod_{j=1}^{n} \mu_{A_{j}^{i}}\left(x_{j}\right)\right)}$

is the fuzzy basis function (FBF). Throughout the paper, it is assumed that the FBFs are selected so that there is always at least one active rule (Wang, 1994), i.e. $\sum_{i=1}^{m}\left(\prod_{j=1}^{n} \mu_{A_{j}^{i}}\left(x_{j}\right)\right)>0$.

It is worth noting that the fuzzy system (22) is commonly used in control applications. Following the universal approximation results (Wang, 1994), the fuzzy system (22) is able to approximate any nonlinear smooth function $f(\underline{x})$ on a compact operating space to an arbitrary degree of accuracy. Of particular importance, it is assumed that the structure of the fuzzy system (i.e. the pertinent inputs, the number of membership functions for each input and the number of rules) and the membership function parameters are properly specified beforehand. The consequent parameters, i.e. $\theta$, are determined by adaptation algorithms.

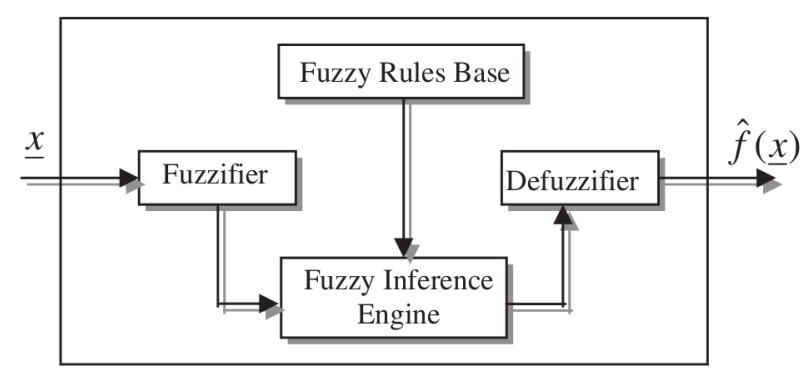

Fig. 1. The basic configuration of a fuzzy logic system.

\subsection{Input nonlinearity}

The mathematical model of the input nonlinearity $\Phi_{i}\left(u_{i}\right)$ is described by

$\Phi_{i}\left(u_{i}\right)= \begin{cases}\phi_{i_{+}}\left(u_{i}\right)\left(u_{i}-u_{i+}\right), & u_{i}>u_{i+}, \\ 0, & -u_{i-} \leqslant u_{i} \leqslant u_{i+}, \\ \phi_{i_{-}}\left(u_{i}\right)\left(u_{i}+u_{i_{-}}\right), & u_{i}<-u_{i_{-}},\end{cases}$

where $\phi_{i+}\left(u_{i}\right)>0$ and $\phi_{i-}\left(u_{i}\right)>0$ are nonlinear functions of $u_{i}$, and $u_{i^{+}}>0, u_{i_{-}}>0$ are constants.

The input nonlinearity $\Phi_{i}\left(u_{i}\right)$ satisfies the following important properties:

$\left(u_{i}-u_{i_{+}}\right) \Phi_{i}\left(u_{i}\right) \geqslant m_{i+}^{*}\left(u_{i}-u_{i+}\right)^{2}, \quad u_{i}>u_{i+}$,

$\left(u_{i}+u_{i-}\right) \Phi_{i}\left(u_{i}\right) \geqslant m_{i-}^{*}\left(u_{i}+u_{i-}\right)^{2}, \quad u_{i}<-u_{i-}$,

where $m_{i+}^{*}$ and $m_{i-}^{*}$ are constants called "gain reduction tolerances". Let us denote $\eta_{i}=\min \left\{m_{i+}^{*}, m_{i-}^{*}\right\}$.

In order to study the characteristics of this input nonlinearity in the control problems, the following assumptions are made:

\section{Assumption 3}

(a) The gain reduction tolerances, $m_{i+}^{*}$ and $m_{i-}^{*}$, are unknown. Therefore, $\eta_{i}$ is also unknown.

(b) The explicit mathematical expression of $\Phi_{i}\left(u_{i}\right)$ is uncertain, except the properties (25) and constants $u_{i+}$ and $u_{i-}$ (which are assumed to be known).

\section{Remark 2}

(1) It is clear from (24) and (25) that for $u_{i^{+}}=u_{i-}=0$, the input nonlinearity $\Phi_{i}\left(u_{i}\right)$ is reduced to the special sector nonlinear function. Hence, the considered MIMO system with the input nonlinearities (24) is a more general form.

(2) Note that the model (24) has also been used in Yan et al. (2005), Chiang et al. (2007), Chang (2007) and Hung et al. (2008). Compared to our contribution, these papers have the following limitations:

- The considered class of chaotic systems is a simple SISO system with sector nonlinearities and/or dead-zones.

- The gain reduction tolerances $m_{i+}^{*}$ and $m_{i-}^{*}$ or their mini$\operatorname{mum} \eta_{i}=\min \left\{m_{i+}^{*}, m_{i-}^{*}\right\}$ are assumed to be known.

- The upper bounds of uncertainties are required to be known.

\section{Design of the fuzzy adaptive controller}

In this section, a fuzzy adaptive variable-structure control scheme is developed for the class of unknown chaotic MIMO systems described by (2). 
By substituting (2) into (20), the dynamics of $S$ become

$\dot{S}=C_{r}^{T} E+F(x)+G \Phi(u)+D(t)-y_{d}^{(r)}$.

Posing $G_{1}=G^{-1}$ and $D_{g}(t)=G^{-1} D(t)$, we have

$G_{1} \dot{S}=G_{1}\left[C_{r}^{T} E-y_{d}^{(r)}+F(x)\right]+\Phi(u)+D_{g}(t)$.

To facilitate the stability analysis and control design, we rewrite the dynamics of $S$ as follows

$$
\begin{aligned}
G_{1} \dot{S} & =G_{1}\left[C_{r}^{T} E-y_{d}^{(r)}+F(x)\right]+\Phi(u)+D_{g}(t) \\
& =\alpha(x, v)+\Phi(u)+D_{g}(t),
\end{aligned}
$$

where $\quad \alpha(x, v)=\left[\alpha_{1}(x, v), \alpha_{2}(x, v), \ldots, \alpha_{p}(x, v)\right]^{T}=G_{1}[v+F(x)]$ $v=C_{r}^{T} E-y_{d}^{(r)}$

and

Assumption 4. There exists an unknown continuous positive function $\bar{\alpha}_{i}(x)$ such that:

$\left|\alpha_{i}(x, v)\right| \leqslant \eta \bar{\alpha}_{i}(x), \quad \forall x \in \Omega_{x} \subset R^{r}$.

where $\eta=\min _{i}\left\{\eta_{i}\right\}$.

Remark 3. Assumption 4 is not restrictive for the following reasons:

- The upper bound $\eta \bar{\alpha}_{i}(x)$ is assumed to be unknown.

- Since $v$ is a function of $\left(x, x_{d}\right), x_{d} \in L_{\infty}$ and $\alpha_{i}(x, v)$ is a continuous function, therefore such a function (i.e. $\left.\bar{\alpha}_{i}(x)\right)$ always exists.

The unknown continuous nonlinear function $\bar{\alpha}_{i}(x)$ can be approximated, on a compact set $\Omega_{\chi}$, by the fuzzy systems (22) as follows:

$\hat{\bar{\alpha}}_{i}(x, \theta)=\theta_{i}^{T} \psi_{i}(x)$,

where $\psi_{i}(x)$ is the FBF vector, which is fixed a priori by the designer, and $\theta_{i}$ is the adjustable parameter vector of the fuzzy system.

Let us define

$\theta_{i}^{*}=\underset{\theta_{i}}{\arg \min }\left[\sup _{x \in \Omega_{x}}\left|\bar{\alpha}_{i}(x)-\hat{\bar{\alpha}}_{i}\left(x, \theta_{i}\right)\right|\right]$

as the optimal values of $\theta_{i}$.

Note that the optimal values of $\theta_{i}$ are artificial constant quantities introduced only for analysis purposes, and their values are not needed when implementing the controller.

Define

$\tilde{\theta}_{i}=\theta_{i}-\theta_{i}^{*}$

as the parameter estimation error, and

$\varepsilon_{i}(x)=\bar{\alpha}_{i}(x)-\hat{\bar{\alpha}}_{i}\left(x, \theta_{i}^{*}\right)$

is the fuzzy approximation error, where $\hat{\bar{\alpha}}_{i}\left(x, \theta_{i}^{*}\right)=\theta_{i}^{* T} \psi_{i}(x)$.

As in the literature (Boulkroune et al., 2006; Boulkroune et al. 2008; Boulkroune, Tadjine, M'saad, \& Farza, 2008; Boulkroune, M'Saad, Tadjine, \& Farza, 2010; Chang, 2001; Liu \& Zheng, 2009; Roopaei \& Jahromi, 2008; Roopaei et al., 2009; Poursamad \& Markazi, 2009; Wang, 1994; Youcef \& Wahba, 2009), we assume that the used fuzzy systems do not violate the universal approximator property on the compact set $\Omega_{x}$, which is assumed large enough so that the input vector of the fuzzy system remains in $\Omega_{x}$ under closed-loop control system. So it is reasonable to assume that the fuzzy approximation error is bounded for all $x \in \Omega_{x}$, i.e.

$\left|\varepsilon_{i}(x)\right| \leqslant \bar{\varepsilon}_{i}, \quad \forall x \in \Omega_{x}$

where $\bar{\varepsilon}_{i}$ is an unknown constant
From the above analysis, we have

$$
\begin{aligned}
\hat{\bar{\alpha}}_{i}\left(x, \theta_{i}\right)-\bar{\alpha}_{i}(x)= & \hat{\bar{\alpha}}_{i}\left(x, \theta_{i}\right)-\hat{\bar{\alpha}}_{i}\left(x, \theta_{i}^{*}\right)+\hat{\bar{\alpha}}_{i}\left(x, \theta_{i}^{*}\right)-\bar{\alpha}_{i}(x), \\
& =\hat{\bar{\alpha}}_{i}\left(x, \theta_{i}\right)-\hat{\bar{\alpha}}_{i}\left(x, \theta_{i}^{*}\right)-\varepsilon_{i}(x), \\
& =\tilde{\theta}_{i}^{T} \psi_{i}(x)-\varepsilon_{i}(x) .
\end{aligned}
$$

To meet the control objective, a suitable fuzzy adaptive variablestructure controller is proposed as follows:

$u_{i}= \begin{cases}-\rho_{i}(t) \operatorname{sign}\left(S_{i}\right)-u_{i-}, & S_{i}>\mathbf{0}, \\ 0, & S_{i}=\mathbf{0}, \\ -\rho_{i}(t) \operatorname{sign}\left(S_{i}\right)+u_{i+}, & S_{i}<\mathbf{0},\end{cases}$

with $\rho_{i}(t)=k_{0 i}+k_{1 i}\left|S_{i}\right|+\theta_{i}^{T} \psi_{i}(x), \forall i=1, \ldots, p$ and

$\dot{k}_{0 i}=-\gamma_{0 i} \sigma_{0 i} k_{0 i}+\gamma_{0 i}\left|S_{i}\right|, \quad k_{0 i}(0) \geqslant 0$,

$\dot{\theta}_{i}=-\gamma_{1 i} \sigma_{1 i} \theta_{i}+\gamma_{1 i}\left|S_{i}\right| \psi_{i}(x), \quad \theta_{i j}(0) \geqslant 0$,

where $\gamma_{0 i}, \gamma_{1 i}, \sigma_{0 i}, \sigma_{1 i}, k_{1 i}>0$ are design constants, and $k_{0 i}$ and $\theta_{i}$ are the online estimates of the uncertain terms $k_{0 i}^{*}=\bar{\varepsilon}_{i}+\bar{d}_{g i}$ and $\theta_{i}^{*}$, respectively. Note that $\bar{d}_{g i}$ are unknown positive constants which satisfy: $\sum_{i=1}^{p} \bar{d}_{g i}\left|S_{i}\right| \geqslant\left|S^{T} D_{g}(t) / \eta\right|$.

Remark 4. With $k_{0 i}(0) \geqslant 0$ and $\theta_{i}(0) \geqslant 0$, it follows from adaptive laws (34) and (35) that their respective solutions satisfy $k_{0 i}(t) \geqslant 0$ and $\theta_{i}(t) \geqslant 0$, for $t>0$.

Multiplying (28) by $S^{T}$ and using Assumption 4, we have

$$
\begin{aligned}
\frac{1}{\eta} S^{T} G_{1} \dot{S} & =\frac{1}{\eta} S^{T} \alpha(x, v)+\frac{1}{\eta} S^{T} \Phi(u)+\frac{1}{\eta} S^{T} D_{g}(t) \\
& \leqslant \sum_{i=1}^{p}\left|S_{i}\right| \bar{\alpha}_{i}(x)+\frac{1}{\eta} S^{T} \Phi(u)+\frac{1}{\eta} S^{T} D_{g}(t) .
\end{aligned}
$$

From (32) and (36), we get

$$
\begin{aligned}
\frac{1}{\eta} S^{T} G_{1} \dot{S} \leqslant & \sum_{i=1}^{p}\left|S_{i}\right| \bar{\alpha}_{i}(x)+\frac{1}{\eta} S^{T} \Phi(u)+\frac{1}{\eta} S^{T} D_{g}(t) \\
\leqslant & -\sum_{i=1}^{p}\left|S_{i}\right| \tilde{k}_{0 i}-\sum_{i=1}^{p}\left|S_{i}\right| \tilde{\theta}_{i}^{T} \psi_{i}(x)+\sum_{i=1}^{p}\left|S_{i}\right| k_{0 i} \\
& +\sum_{i=1}^{p}\left|S_{i}\right| \theta_{i}^{T} \psi_{i}(x)+\frac{1}{\eta} S^{T} \Phi(u),
\end{aligned}
$$

where $\tilde{\theta}_{i}=\theta_{i}-\theta_{i}^{*}$ and $\tilde{k}_{0 i}=k_{0 i}-k_{0 i}^{*}=k_{0 i}-\bar{\varepsilon}_{i}-\bar{d}_{g i}$.

Theorem 1. Consider the system (2) and suppose that Assumptions 1-4 are satisfied. Then, the control law defined by (33)-(35) guarantees the following properties:

- All signals in the closed loop system are uniformly ultimately bounded.

- The solution of the closed-loop error system exponentially converges to an adjustable region.

Proof of Theorem 1. Let us consider the following Lyapunov function candidate:

$V=\frac{1}{2 \eta} S^{T} G_{1} S+\frac{1}{2} \sum_{i=1}^{p} \frac{1}{\gamma_{0 i}} \tilde{k}_{0 i}^{2}+\frac{1}{2} \sum_{i=1}^{p} \frac{1}{\gamma_{1 i}} \tilde{\theta}_{i}^{T} \tilde{\theta}_{i}$.

Its time derivative is given by

$\dot{V}=\frac{1}{\eta} S^{T} G_{1} \dot{S}+\sum_{i=1}^{p} \frac{1}{\gamma_{0 i}} \tilde{k}_{0 i} \dot{k}_{0 i}+\sum_{i=1}^{p} \frac{1}{\gamma_{1 i}} \tilde{\theta}_{i}^{T} \dot{\theta}_{i}$

with $\dot{\mathrm{G}}_{1}=0$

We can easily show from (33) that $u_{i}<-u_{i-}$ for $S_{i}>0$ and $u_{i}>u_{i+}$ for $S_{i}<0$. Thus, we get from (25) and (33), that for $S_{i}>0$ 
$\left(u_{i}+u_{i-}\right) \Phi_{i}\left(u_{i}\right)=-\rho_{i}(t) \operatorname{sign}\left(S_{i}\right) \Phi_{i}\left(u_{i}\right) \geqslant m_{i-}^{*} \rho_{i}^{2}(t) \geqslant \eta \rho_{i}^{2}(t)$

and for $S_{i}<0$

$\left(u_{i}-u_{i+}\right) \Phi_{i}\left(u_{i}\right)=-\rho_{i}(t) \operatorname{sign}\left(S_{i}\right) \Phi_{i}\left(u_{i}\right) \geqslant m_{i+}^{*} \rho_{i}^{2}(t) \geqslant \eta \rho_{i}^{2}(t)$.

Then, for $S_{i}<0$ and $S_{i}>0$, we have

$-\rho_{i}(t) \operatorname{sign}\left(S_{i}\right) \Phi_{i}\left(u_{i}\right) \geqslant \eta \rho_{i}^{2}(t)$.

From (42) and using the facts $S_{i}^{2}>0$ and $S_{i} \operatorname{sign}\left(S_{i}\right)=\left|S_{i}\right|$, we have

$-\rho_{i}(t) S_{i}^{2} \operatorname{sign}\left(S_{i}\right) \Phi_{i}\left(u_{i}\right) \geqslant \eta \rho_{i}^{2}(t) S_{i}^{2}=\eta \rho_{i}^{2}(t)\left|S_{i}\right|^{2}$.

Finally for all $S_{i}$ (i.e. for $S_{i}<0, S_{i}=0$ and $S_{i}>0$ ), we have

$S_{i} \Phi_{i}\left(u_{i}\right) \leqslant-\eta \rho_{i}(t)\left|S_{i}\right|$

Using the expressions (34), (35), (44) and (37), (39) becomes

$$
\begin{aligned}
\dot{V} & \leqslant \sum_{i=1}^{p}\left|S_{i}\right| k_{0 i}+\sum_{i=1}^{p}\left|S_{i}\right| \theta_{i}^{T} \psi_{i}(x)+\frac{1}{\eta} \sum_{i=1}^{p} S_{i} \Phi_{i}\left(u_{i}\right)-\sum_{i=1}^{p} \sigma_{0 i} \tilde{k}_{0 i} k_{0 i} \\
& -\sum_{i=1}^{p} \sigma_{1 i} \tilde{\theta}_{i}^{T} \theta_{i} \leqslant \sum_{i=1}^{p}\left|S_{i}\right| k_{0 i}+\sum_{i=1}^{p}\left|S_{i}\right| \theta_{i}^{T} \psi_{i}(x)+\sum_{i=1}^{p}-\rho_{i}(t)\left|S_{i}\right| \\
& -\sum_{i=1}^{p} \sigma_{0 i} \tilde{k}_{0 i} k_{0 i}-\sum_{i=1}^{p} \sigma_{1 i} \tilde{\theta}_{i}^{T} \theta_{i} \\
& =-\sum_{i=1}^{p} k_{1 i} S_{i}^{2}-\sum_{i=1}^{p} \sigma_{0 i} \tilde{k}_{0 i} k_{0 i}-\sum_{i=1}^{p} \sigma_{1 i} \tilde{\theta}_{i}^{T} \theta_{i} .
\end{aligned}
$$

We can easily verify that

$$
\begin{aligned}
& -\sigma_{0 i} \tilde{k}_{0 i} k_{0 i} \leqslant-\frac{\sigma_{0 i}}{2} \tilde{k}_{0 i}^{2}+\frac{\sigma_{0 i}}{2} k_{0 i}^{* 2} \\
& -\sigma_{1 i} \tilde{\theta}_{i}^{T} \theta_{i} \leqslant-\frac{\sigma_{1 i}}{2}\left\|\tilde{\theta}_{i}\right\|^{2}+\frac{\sigma_{1 i}}{2}\left\|\theta_{i}^{*}\right\|^{2} .
\end{aligned}
$$

Using the previous inequalities, (45) becomes

$\dot{V} \leqslant-\sum_{i=1}^{p} k_{1 i} S_{i}^{2}-\sum_{i=1}^{p} \frac{\sigma_{0 i}}{2} \tilde{k}_{0 i}^{2}-\sum_{i=1}^{p} \frac{\sigma_{1 i}}{2}\left\|\tilde{\theta}_{i}\right\|^{2}+\sum_{i=1}^{p} \frac{\sigma_{0 i}}{2} k_{0 i}^{* 2}+\sum_{i=1}^{p} \frac{\sigma_{1 i}}{2}\left\|\theta_{i}^{*}\right\|^{2}$.

Since $G \geqslant \sigma_{g 0} I_{p}$ (recall that the matrix $G$ is symmetric and positivedefinite), than

$S^{T} G^{-1} S=S^{T} G_{1} S \leqslant \frac{1}{\sigma_{g 0}}\|S\|^{2}$.

From (46) and (47), we have

$\dot{V} \leqslant-\mu V+\pi$,

where $\pi=\sum_{i=1}^{p} \frac{\sigma_{0 i}}{2} k_{0 i}^{* 2}+\sum_{i=1}^{p} \frac{\sigma_{1 i}}{2}\left\|\theta_{i}^{*}\right\|^{2}$

$\mu=\min \left\{\min _{i}\left\{2 \eta \sigma_{g 0} k_{1 i}\right\}, \min _{i}\left\{\gamma_{0 i} \sigma_{0 i}\right\}, \min _{i}\left\{\gamma_{1 i} \sigma_{1 i}\right\}\right\}$.

Multiplying (48) by $e^{\mu t}$ yields

$\frac{d}{d t}\left(V e^{\mu t}\right) \leqslant \pi e^{\mu t}$

Integrating (49) over $[0, t]$, we have

$0 \leqslant V(t) \leqslant \frac{\pi}{\mu}+\left(V(0)-\frac{\pi}{\mu}\right) e^{-\mu t}$.

Therefore, all signals of the closed-loop system, i.e. $k_{0 i}, \theta_{i}, S_{i}, E$ and $x$, are uniformly ultimately bounded. Then, $u_{i}$ is also bounded.

From (38), $V(0)$ can be determined as follows

$$
\begin{aligned}
V(0)= & \frac{1}{2 \eta} S(0)^{T} G_{1} S(0)+\frac{1}{2} \sum_{i=1}^{p} \frac{1}{\gamma_{0 i}}\left(k_{0 i}(0)-k_{0 i}^{*}\right)^{2}+\frac{1}{2} \sum_{i=1}^{p} \\
& \times \frac{1}{\gamma_{1 i}}\left(\theta_{i}(0)-\theta_{i}^{*}\right)^{T}\left(\theta_{i}(0)-\theta_{i}^{*}\right) .
\end{aligned}
$$

Since $G_{1}$ is symmetric and positive-definite (i.e. it exists an unknown positive constant $\sigma_{g 1}$, such that: $G_{1} \geqslant \sigma_{g 1} I_{p}$ ), from (50) and (38), we have

$\left|S_{i}\right| \leqslant\left(\frac{2 \eta}{\sigma_{g 1}}\left(\frac{\pi}{\mu}+\left(V(0)-\frac{\pi}{\mu}\right) e^{-\mu t}\right)\right)^{1 / 2}$

Then, the solution of $S_{i}$ exponentially converges to a bounded region $\Omega_{S_{i}}=\left\{S_{i} \| S_{i} \mid \leqslant\left(\frac{2 \eta}{\sigma_{g 1}} \frac{\pi}{\mu}\right)^{1 / 2}\right\}$. This ends the proof of the theorem.

Remark 5 If $\Phi_{i}\left(u_{i}\right)=u_{i}$ (or when $u_{i_{+}}=u_{i-}=0$, and $\phi_{i+}\left(u_{i}\right)=$ $\left.\phi_{i-}\left(u_{i}\right)=1\right)$, i.e. there are no both dead-zones and sector nonlinear ities in the input function, we can show that the proposed controller remains still applicable to such MIMO chaotic systems.

\section{Remark 6}

(1) In the case where $u_{i^{+}}=u_{i-}=u_{i 0}$, (33) is reduced to the following expression:

$u_{i}=-\left(\rho_{i}(t)+u_{i 0}\right) \operatorname{sign}\left(S_{i}\right)$,

where $\rho_{i}(t)=k_{0 i}+k_{1 i}\left|S_{i}\right|+\theta_{i}^{T} \psi_{i}(x)$.

(2) To eliminate the chattering effect caused by the discontinuous control term in (33) and (52), the function sign(.) must be replaced by any equivalent smooth function such as: tanh (.), $\arctan ()$, Sat(.). ..etc.

\section{Simulation results}

In this section, simulation studies are carried out to show the effectiveness of the proposed adaptive fuzzy controller. For this end, we consider the tracking control problem of the uncertain unified-chaotic system. This unified-chaotic system introduced by Lü et al. (2002) is described by the following dynamics (Ablay, 2009):

$$
\left\{\begin{array}{l}
\dot{x}_{1}=(25 \alpha+10)\left(x_{2}-x_{1}\right) \\
\dot{x}_{2}=(28-35 \alpha) x_{1}+(29 \alpha-1) x_{2}-x_{1} x_{3} \\
\dot{x}_{3}=x_{1} x_{2}-(8+\alpha) x_{3} / 3
\end{array}\right.
$$

where $x_{1}, x_{2}, x_{3}$ are state variables and $\alpha \in[0,1]$ is the system parameter. When $\alpha \in[0,0.8[$, system (53) is called the generalized Lorenz chaotic system (Lorenz, 1963). When $\alpha=0.8$, system (53) is called the Lü chaotic system (Lü \& Chen, 2002), and when $\alpha \in] 0.8,1]$, system (53) is called the generalized Chen chaotic system (Chen \& Ueta, 1999). It is worth noting that the unified chaotic system can be seen in atmospheric sciences, laser devices, and other systems related to convection (Ablay, 2009).

The unified-chaotic system with control inputs and externaldisturbances is described by:

$\left\{\begin{array}{l}\dot{x}_{1}=(25 \alpha+10)\left(x_{2}-x_{1}\right)+\Phi_{1}\left(u_{1}\right)+d_{1}(t) \\ \dot{x}_{2}=(28-35 \alpha) x_{1}+(29 \alpha-1) x_{2}-x_{1} x_{3}+\Phi_{2}\left(u_{2}\right)+d_{2}(t) \\ \dot{x}_{3}=x_{1} x_{2}-(8+\alpha) x_{3} / 3+\Phi_{3}\left(u_{3}\right)+d_{3}(t)\end{array}\right.$

where $\Phi_{i}\left(u_{i}\right), i=1,2,3$, are the models of the inevitable input nonlinearities.

Let $y=x=\left[x_{1}, x_{2}, x_{3}\right]^{T}, u=\left[u_{1}, u_{2}, u_{3}\right]^{T}, \Phi(u)=\left[\Phi_{1}\left(u_{1}\right), \Phi_{2}\left(u_{2}\right)\right.$, $\left.\Phi_{3}\left(u_{3}\right)\right]^{T}$, and $D(t)=\left[\begin{array}{lll}d_{1}(t), & d_{2}(t), d_{3}(t)\end{array}\right]^{T}$. Then, the system (eq. (54)) can be expressed as follows:

$\dot{y}=F(x)+G \Phi(u)+D(t)$, 

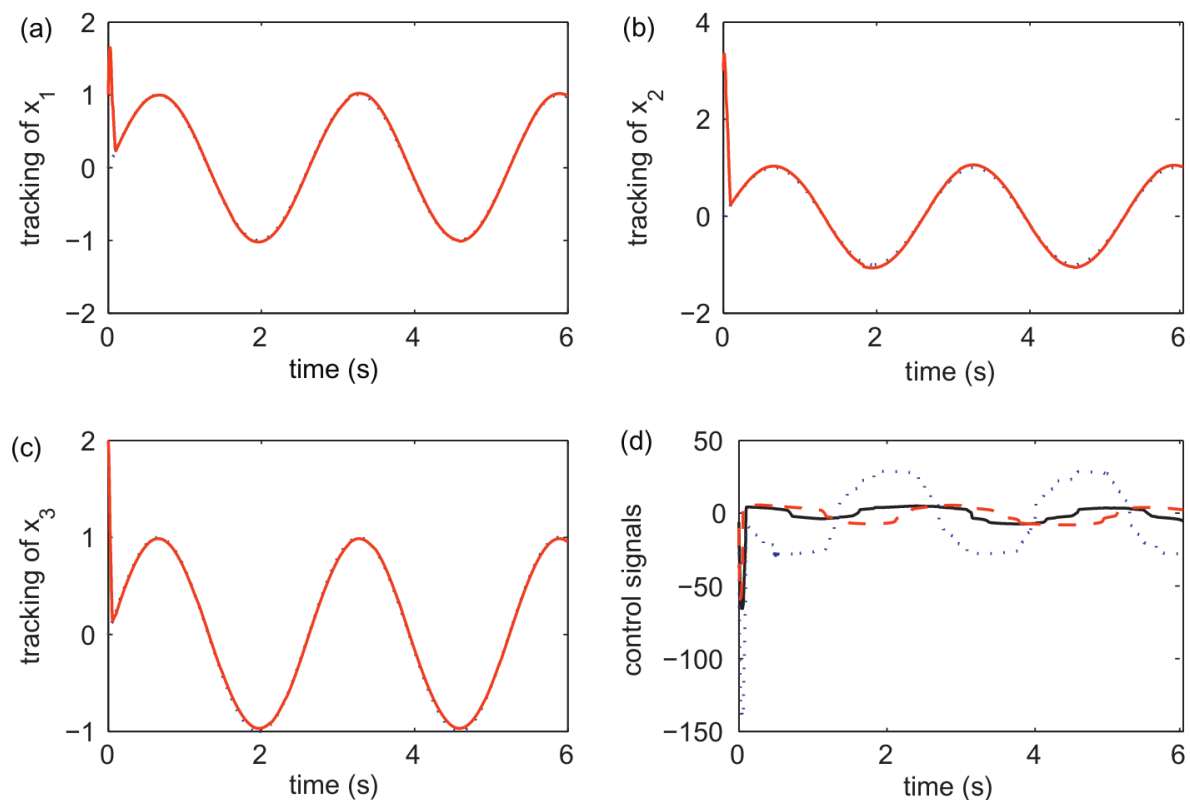

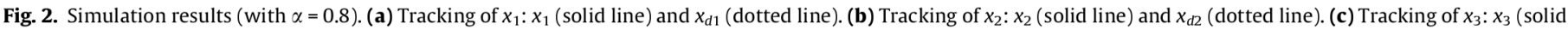
line) and $x_{d 3}$ (dotted line). (d) Control input signals applied at $t=0 s: u_{1}$ (solid line), $u_{2}$ (dotted line) and $u_{3}$ (dashed line).
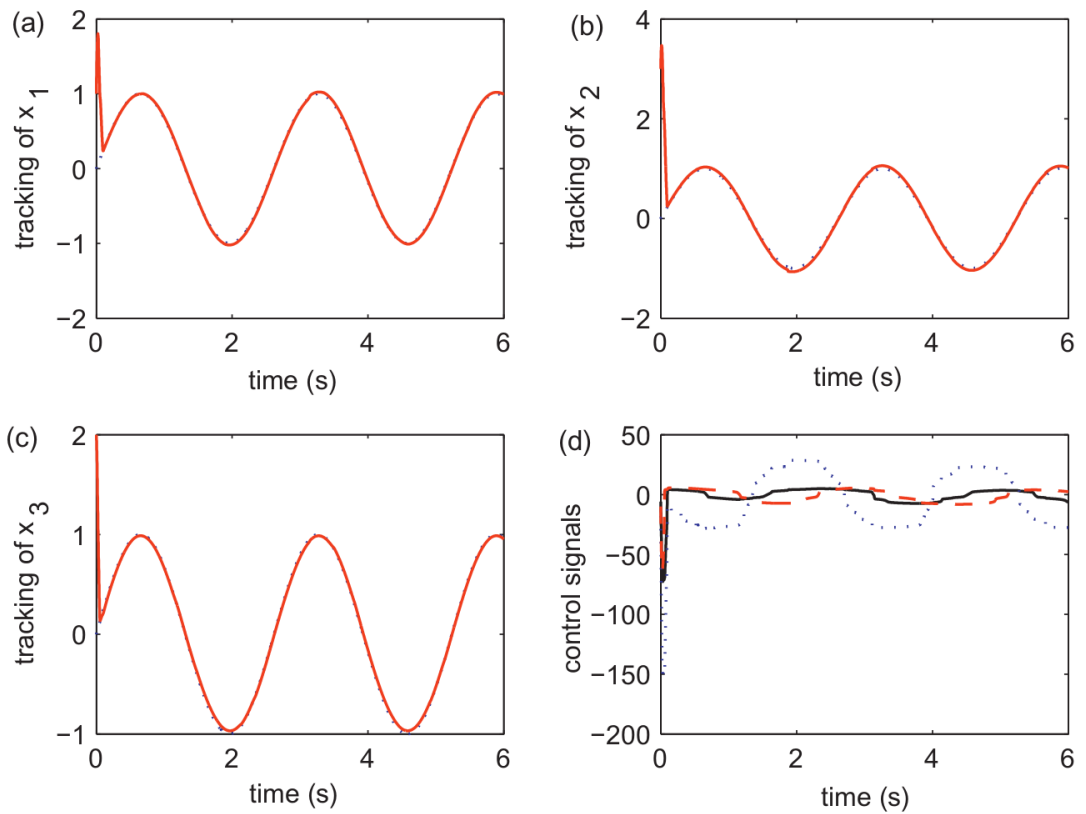

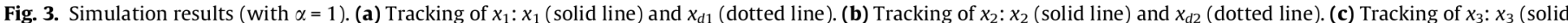
line) and $x_{d 3}$ (dotted line). (d) Control input signals applied at $t=0 s: u_{1}$ (solid line), $u_{2}$ (dotted line) and $u_{3}$ (dashed line).

where

$F(x)=\left(\begin{array}{c}(25 \alpha+10)\left(x_{2}-x_{1}\right) \\ (28-35 \alpha) x_{1}+(29 \alpha-1) x_{2}-x_{1} x_{3} \\ x_{1} x_{2}-(8+\alpha) x_{3} / 3\end{array}\right), \quad G=\left(\begin{array}{lll}1 & 0 & 0 \\ 0 & 1 & 0 \\ 0 & 0 & 1\end{array}\right)$

The input nonlinearities, $\Phi_{i}\left(u_{i}\right)$, for $i=1,3$, are described by:

$\Phi_{i}\left(u_{i}\right)= \begin{cases}\left(u_{i}-2\right)\left(1-0.3 \sin \left(u_{i}\right)\right), & u_{i}>2, \\ 0, & -2 \leqslant u_{i} \leqslant 2, \\ \left(u_{i}+2\right)\left(0.8-0.3 \cos \left(u_{i}\right)\right), & u_{i}<-2,\end{cases}$
But, the input nonlinearity $\Phi_{2}\left(u_{2}\right)$ is supposed to be:

$\Phi_{2}\left(u_{2}\right)= \begin{cases}\left(u_{2}-5\right)\left(1-0.3 \sin \left(u_{2}\right)\right), & u_{2}>5, \\ 0, & -5 \leqslant u_{2} \leqslant 5, . \\ \left(u_{2}+5\right)\left(0.8-0.3 \cos \left(u_{2}\right)\right), & u_{2}<-5,\end{cases}$

It is worth noting that the system model (i.e. $F(x), G, \Phi(u)$ and $D(t)$ ) is assumed to be unknown by the controller, except some structural properties such as:

- The symmetry and the sign of $G$,

- The properties (25) of $\Phi_{i}\left(u_{i}\right)$ and the knowledge of $u_{i_{+}}$and $u_{i_{-}}$, with $i(=1,2,3)$. 
In fact, the model (54) is only required here for simulation purposes.

The initial conditions of the system are taken as $x(0)=\left[x_{1}(0), x_{2}(0), x_{3}(0)\right]^{T}=[1,3,2]^{T}$. The desired trajectories are selected as $x_{d 1}=x_{d 2}=x_{d 3}=\sin (2.4 t)$. The external disturbances are selected as follows: $d_{1}(t)=d_{2}(t)=d_{3}(t)=\sin (t)$.

The adaptive fuzzy systems, $\theta_{i}^{T} \psi_{i}(x)$, with $i=1,2,3$, have the vector $x=\left[x_{1}, x_{2}, x_{3}\right]^{T}$ as input. For each entry variable of these fuzzy systems, as in Boulkroune et al. (2008), we define three triangular membership functions uniformly distributed on the intervals $[-2,2]$. The design parameters are chosen as follows: $\gamma_{01}=\gamma_{03}=$ $30, \gamma_{02}=80, \gamma_{11}=\gamma_{12}=\gamma_{13}=4000, \quad \sigma_{01}=\sigma_{02}=\sigma_{03}=0.001, \quad \sigma_{11}$ $=\sigma_{12}=\sigma_{13}=0.0005, \lambda_{1}=\lambda_{2}=\lambda_{3}=2, k_{11}=k_{12}=k_{13}=2$. The initial conditions are selected as: $k_{01}(0)=k_{02}(0)=k_{03}(0)=0, \theta_{1 j}(0)=\theta_{2 j}$ $(0)=\theta_{3 j}(0)=0, j=1, \ldots, 27$.

Note that, in all simulations, the discontinuous function $\operatorname{sign}\left(S_{i}\right)$ has been replaced by a smooth function $\tanh \left(k_{s i} S_{i}\right)$, with $k_{s i}=20$, $i=1,2,3$.

According to the value of $\alpha$, we will consider two simulation cases:

- $\alpha=0.8$, the proposed controller is applied to Lü chaotic system,

- $\alpha=1$, the proposed controller is applied to the generalized Chen chaotic system.

(a) Case 1 (when $\alpha=0.8$ ) The simulation results of the proposed controller applied at time $t=0 \mathrm{~s}$ are shown in Fig. 2. These simulation results clearly show that the tracking performance is nice. Consequently the effects due to system uncertainties, unavoidable input nonlinearities (dead-zone and sector nonlinearity) and bounded external disturbances in Lü chaotic system can be efficiently diminished by the proposed fuzzy adaptive control scheme. From this figure, we can also see the boundedness of the control signals.

(b) Case 2 (when $\alpha=1$ ) The simulation results of this case are shown in Fig. 3. From these simulation results, we can see that the states $\left(x_{1}, x_{2}, x_{3}\right)$ quickly converge to respective desired trajectories $\left(x_{d 1}, x_{d 2}, x_{d 3}\right)$ by activation of control signals at time $t=0 \mathrm{~s}$.

\section{Conclusion}

In this paper, a fuzzy adaptive variable-structure controller for a class of MIMO unknown chaotic systems subject to actuator sector nonlinearities and dead-zones has been proposed. An adaptive fuzzy system has been used to reasonably approximate the upper bounds of the uncertainties. Of fundamental interest, it has been proven by using a Lyapunov approach that the proposed control system is stable and its underlying tracking error converges exponentially to an adjustable region. Simulation results have been given to emphasize the effectiveness of the proposed controller.

\section{References}

Ablay, G. (2009). Sliding mode control of uncertain unified chaotic systems. Nonlinear Analysis: Hybrid systems. doi:10.1016/j.nahs.2009.04.002.

Boulkroune, A., Chekireb, H., Tadjine, M., \& Bouatmane, S. (2006). Observer-based adaptive feedback controller of a class of chaotic systems. International Journal of Bifurcation and Chaos, 16(2), 189-196.

Boulkroune, A., Chekireb, H., Tadjine, M., \& Bouatmane, S. (2007). An adaptive feedback controller with observer for linearizable chaotic systems. Control and Intelligent Systems, 35(2), 1-8.

Boulkroune, A., Chekireb, H., Tadjine, M., \& M'Saad, M. (2006). Fuzzy adaptive controller based on observers for a class of unknown chaotic systems. In First
IFAC Conference on Analysis and Control of Chaotic Systems, Reims-France, June $28-30$

Boulkroune, A., M'Saad, M., Tadjine, M., \& Farza, M. (2008). Adaptive fuzzy control for MIMO nonlinear systems with unknown dead-zone. In Proceedings of fourth International IEEE Conference on Intelligent Systems, Varna, Bulgaria (pp. 450455).

Boulkroune, A., M'Saad, M., Tadjine, M., \& Farza, M. (2010). Fuzzy adaptive controller for MIMO nonlinear systems with known and unknown control direction, Fuzzy sets and systems doi:10.1016/j.fss.2009.04.011.

Boulkroune, A., Tadjine, M., M'saad, M., \& Farza, M. (2008). How to design a fuzzy adaptive control based on observers for uncertain affine nonlinear systems. Fuzzy Sets and Systems, 159, 926-948.

Chang, Y. C. (2001). A robust tracking control for chaotic Chua's circuits via fuzzy approach. IEEE Transactions on Circuits Systems I: Fundamental Theory and Applications, 48(7), 889-895.

Chang, K. M. (2007). Adaptive control for a class of chaotic systems with nonlinear inputs and disturbances. Chaos, Solitons E Fractals, 36, 460-468.

Chen, G., \& Dong, X. (1998). From chaos to order: Methodologies. Perspectives and applications. Singapore: World-Scientific.

Chen, C. H., Lin, C. M., \& Chen, T. Y. (2008). Intelligent adaptive control for MIMO uncertain nonlinear systems. Expert Systems with Applications, 35, 865-877.

Chen, G. R., \& Ueta, T. (1999). Yet another chaotic attractor. International Journal of Bifurcation and Chaos, 9, 146-156.

Chiang, T. Y., Hung, M. L., Yan, J. J., Yang, Y. S., \& Chang, J. F. (2007). Sliding mode control for uncertain unified chaotic systems with input nonlinearity. Chaos, Solitons E' Fractals, 34, 437-442.

Feki, M. (2003). An adaptive feedback controller for linearizable chaotic systems Chaos, Solitons \&' Fractals, 15, 883-890.

Ge, S. S., \& Wang, C. (2000). Adaptive control of uncertain Chua's circuits. IEEE Transactions on Circuits Systems I: Fundamental Theory and Applications, 47(9), $1397-1402$

Ge, S. S., \& Wang, C. (2002). Uncertain chaotic system control via adaptive neural design. International Journal of Bifurcation and Chaos, 12(5), 1097-1109.

Ghezi, L. L., \& Peccardi, C. (1997). PID control of a chaotic system: An application to an epidemiological model. Automatica, 33, 181-191.

Hsu, K.-C., Wang, W.-Y., \& Lin, P.-Z. (2004). Sliding mode control for uncertain nonlinear systems with multiple inputs containing sector nonlinearities and dead-zones. IEEE Transactions on Systems, Man and Cybernetic, Part-B, 34(1), 374-380.

Hua, C. C., Guan, X. P., \& Shi, P. (2005). Adaptive feedback control for a class of chaotic systems. Chaos, Solitons \& Fractals, 23, 757-765.

Hung, Y. C., Yan, J. J., \& Liao, T. L. (2008). Projective synchronization of Chua's chaotic systems with dead-zone in the control input. Mathematics and computers in Simulation, 77, 374-382.

Kar, I., \& Behera, L. (2009). Direct adaptive neural control for affine nonlinear systems. Applied soft computing, 9, 756-764.

Liu, Y. J., \& Zheng, Y. Q. (2009). Adaptive robust fuzzy control for a class of uncertain chaotic systems. Nonlinear dynamics, 57(3), 431-439.

Lorenz, E. N. (1963). Deterministic nonperiodic flow. Journal of the Atmospheric Science, 20, 130-141.

Lü, J. H., \& Chen, G. R. (2002). A new chaotic attractor coined. International Journal of Bifurcation and Chaos, 12(3), 659-661.

Lü, J. H., Chen, G. R., Cheng, D. Z., \& Celikovssky, S. (2002). Bridge the gap between the Lorenz and Chen system. International Journal of Bifurcation and Chaos, 12 , 2917-2926.

Nazzal, J. M., \& Natsheh, A. N. (2007). Chaos control using sliding-mode theory. Chaos, Solitons \& Fractals, 33, 695-702.

Ott, E., Grebogi, C., \& Yorke, J. A. (1990). Controlling chaos. Physical Review Letters, 64, 1196-1199.

Poursamad, A., \& Markazi, A. H. D. (2009). Adaptive fuzzy sliding-mode control for multi-input multi-output chaotic systems. Chaos, Solitons \& Fractals, 42(5), 3100-3109.

Roopaei, M., \& Jahromi, M. Z. (2008). Synchronisation of two different chaotic systems using novel adaptive fuzzy sliding mode control. Chaos, 18, 033133.

Roopaei, M., Jahromi, M. Z., \& Jafari, S. (2009). Adaptive gain fuzzy sliding mode control for the synchronisation of nonlinear chaotic gyros. Chaos, 19, 013125.

Vanecek, A., \& Celikovsky, S. (1996). Control systems: From linear analysis and to synthesis of chaos. London: Prentice-Hall.

Wang, L. X. (1994). Adaptive fuzzy systems and control: Design and stability analysis. Englewood Cliffs, NJ: Prentice-Hall.

Wang, C., \& Ge, S. S. (2001). Adaptive backstepping control of uncertain Lorenz system. International Journal of Bifurcation and Chaos, 11(4), 1115-1119.

Yan, J. J., Shyu, K. K., \& Lin, J. S. (2005). Adaptive variable structure control for uncertain chaotic systems containing dead-zone nonlinearity. Chaos, Solitons $\mathcal{E}$ Fractals, 25, 347-355.

Yau, H. T., Chen, C. K., \& Chen, C. L. (2000). Sliding mode control of chaotic systems with uncertainties. International Journal of Bifurcation and Chaos, 10, 1139-1147.

Youcef, H. A., \& Wahba, M. A. (2009). Adaptive fuzzy mimo control of induction motors. Expert Systems with Applications, 36, 4171-4175. 